\title{
CHARGE EXCHANGE AVALANCHE AT THE COMETOPAUSE
}

\author{
Tamas I. Gombosi ${ }^{1}$
}

Space Physics Research Laboratory, Department of Atmospheric and Oceanic Science The University of Michigan, Ann Arbor, MI 48109

Abstract. A sharp ( $\leq 10^{4} \mathrm{~km}$ thick) transition from a solar wind proton dominated flow to a plasma population primarily consisting of relatively cold cometary heavy ions has been observed at a cometocentric distance of about $1.6 \times 10^{5} \mathrm{~km}$ by the VEGA and GIOTTO missions. This boundary (the cometopause) was thought to be related to charge transfer processes, but its location and thickness are inconsistent with conventionally estimated ion - neutral coupling boundaries. In this paper a two-fluid model is used to investigate the major physical processes at the cometopause. By adopting observed comet Halley parameters the model is able to reproduce the location and the thickness of this charge exchange boundary.

\section{Introduction}

The cometopause was first observed by the VEGA-2 spacecraft during its inbound pass (Gringauz et al. 1986). Plasma observations in the coma of comet Halley indicated that around $1.6 \times 10^{5} \mathrm{~km}$ from the nucleus a sharp boundary (the cometopause) separates the solar wind controlled external and the heavy cometary ion dominated internal regions (Gringauz et al. 1986, Balsiger et al. 1986, Reme et al. 1986). The observations were interpreted in terms of ion - neutral collisional coupling (Gringauz et al. 1986), but the details of this process have not been really understood. The main difficulty was to explain the location and the surprisingly small thickness of the cometopause: the ion - neutral collisional boundary was expected to be at about $1.5 \times 10^{4} \mathrm{~km}$ from the nucleus, much closer than that which has been observed (cf. Mendis et al. 1986, Galeev 1986).

In this paper a two-fluid (solar wind protons and cometary heavy ions) steady-state model is used in order to describe the shocked plasma flow along the subsolar flow line. It is assumed that the proton population follows a Maxwellian velocity distribution, while the cometary ion velocity distribution is isotropic in the plasma frame of reference. Charge exchange and photoionization are considered as the dominant sources. This model is basically a two-fluid generalization of an earlier single-fluid model developed by Galeev et al. (1985).

\section{Basic assumptions and governing equations}

In the present work a two fluid steady-state model of the shocked, subsolar cometary plasma flow through an expanding cometary atmosphere (dominated by a single heavy neutral species) is considered. It is assumed that the implanted cometary ions have an isotropic pitch-angle distribution in the decelerating plasma frame of reference, while the solar wind particles follow a Maxwellian velocity distribution. Solar wind ions are lost by charge exchange; cometary ions are created by photoionization and charge transfer and lost by charge exchange with heavy cometary neutrals. The moments

${ }^{1}$ Also at Central Research Institute for Physics, Budapest, Hungary

Copyright 1987 by the American Geophysical Union.

Paper number 7L6658D

0094-8276/87/007L-6658\$03.00 of the steady-state solar wind transport equation are the following (cf. Gombosi 1987):

$$
\begin{gathered}
\vec{\nabla} \cdot\left(n_{s w} \vec{u}\right)=-\sigma_{p n} n_{n} I_{s w}^{(0)} \\
n_{s w}(\vec{u} \cdot \vec{\nabla}) \vec{u}=-\frac{1}{m_{s w}} \vec{\nabla} p_{s w}-\vec{u} \sigma_{p n} n_{n} I_{s w}^{(1)} \\
(\vec{u} \cdot \vec{\nabla}) p_{s w}=-\frac{5}{3} p_{s w}(\vec{\nabla} \cdot \vec{u})-m_{s w} \frac{u^{2}}{3} \sigma_{p n} n_{n} I_{s w}^{(2)}
\end{gathered}
$$

The moments of the implanted ion transport equation yield the following conservation equations (cf. Gombosi 1987):

$$
\begin{gathered}
\vec{\nabla} \cdot\left(n_{i} \vec{u}\right)=u S_{i} \\
n_{i}(\vec{u} \cdot \vec{\nabla}) \vec{u}=-\frac{1}{m_{i}} \vec{\nabla} p_{i}-\vec{u}\left[u S_{i}+n_{n} \sigma_{i n}\left(I_{i}^{(0)}+I_{i}^{(1)}\right)\right] \\
(\vec{u} \cdot \vec{\nabla}) p_{i}=-\frac{5}{3} p_{i}(\vec{\nabla} \cdot \vec{u})+\frac{m_{i} u^{2}}{3}\left[u S_{i}+n_{n} \sigma_{i n}\left(I_{i}^{(0)}-I_{i}^{(2)}\right)\right]
\end{gathered}
$$

In equations (1) through (6) $n_{n}$ is the cometary neutral number density, $\sigma_{\mathrm{pn}}$ and $\sigma_{\text {in }}$ are the proton - cometary neutral and implanted ion - cometary neutral charge transfer cross sections, and $u$ is the plasma flow velocity, while $n_{s w}, n_{1}$, $\mathrm{m}_{\mathrm{sw}}, \mathrm{m}_{\mathrm{i}}, \mathrm{p}_{\mathrm{sw}}$, and $\mathrm{p}_{\mathrm{i}}$ denote the solar wind and implanted ion number densities, masses, and pressures, respectively. The cometary ion source term, $S_{i}$, and the charge exchange loss functions, $I^{(k)}$, are the following:

$$
\begin{gathered}
S_{i}=\frac{n_{n}}{u}\left(\frac{1}{\tau}+\sigma_{p n} I_{s w}^{(0)}\right) \\
I^{(0)}=\int d \Omega_{v} \int_{0}^{\infty} d v v^{2}|\vec{u}+\vec{v}| f(\vec{x}, v) \\
I^{(1)}=\int d \Omega_{v} \int_{0}^{\infty} d v v^{2} \frac{\vec{u} \cdot \vec{v}}{u^{2}}|\vec{u}+\vec{v}| f(\vec{x}, v) \\
I^{(2)}=\int d \Omega_{v} \int_{0}^{\infty} d v v^{2} \frac{v^{2}}{u^{2}}|\vec{u}+\vec{v}| f(\vec{x}, v)
\end{gathered}
$$

where $\tau_{\mathrm{n}}$ is the photoionization lifetime of cometary neutrals, while $f(x, v)$ is the phase space distribution function in the plasma frame of reference. It can be shown that in the case of a Maxwellian velocity distribution the loss functions become

$$
I^{(0)}=u n\left[\left(1+\frac{1}{2 s^{2}}\right) \operatorname{erf}(s)+\frac{e^{-s^{2}}}{s \sqrt{\pi}}\right]
$$


SHOCKED FLOW ALONG THE SUBSOLAR FLOW LINE
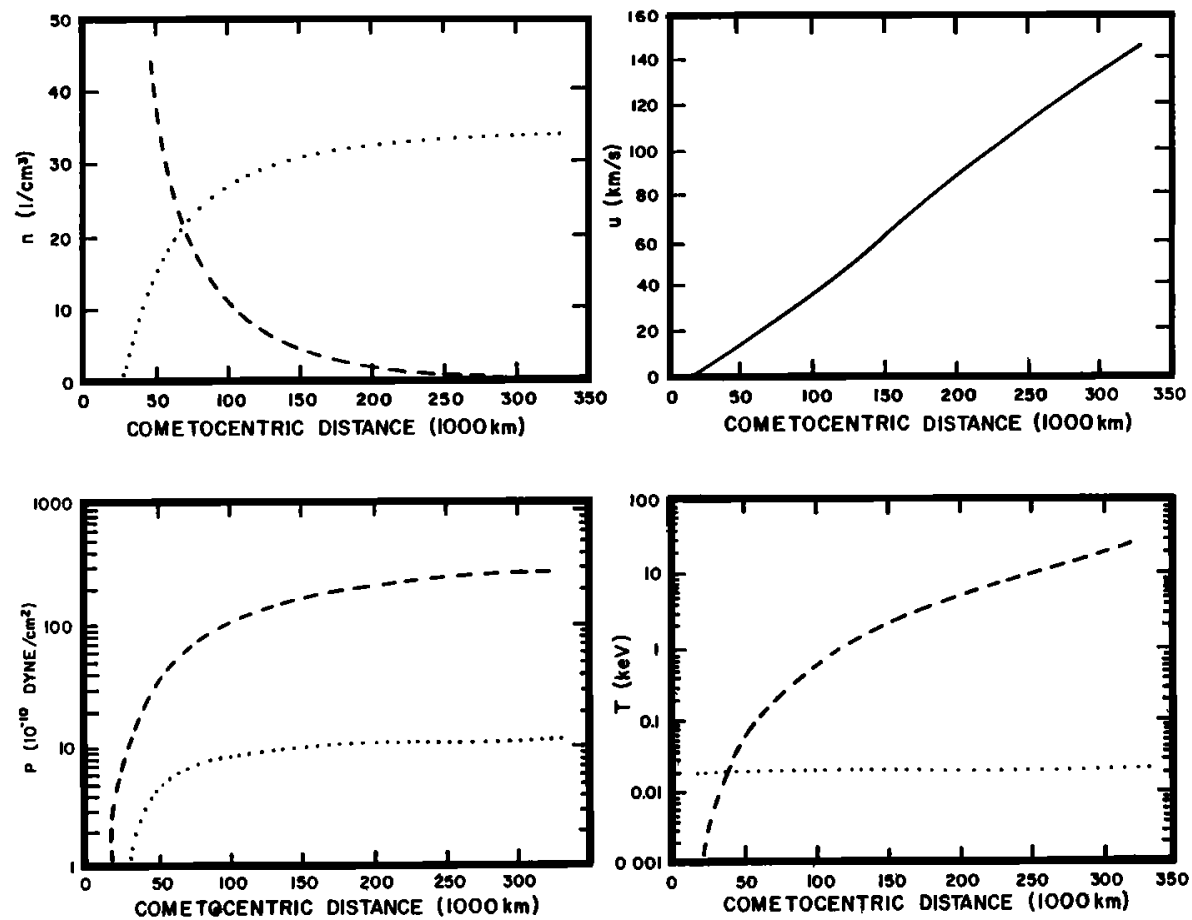

Figure 1. Proton and cometary ion flow parameters along the subsolar flow line. Dashed lines represent solar wind protons, while dotted lines denote cometary ions. The four panels represent number densities, flow velocity, pressures and temperatures, respectively.

$$
\begin{aligned}
& I^{(1)}=\frac{p}{u m}\left[\left(1-\frac{1}{2 s^{2}}\right) \operatorname{erf}(s)+\frac{e^{-s^{2}}}{s \sqrt{\pi}}\right] \\
& I^{(2)}=\frac{3 p}{u m}\left[\left(1+\frac{5}{6 s^{2}}\right) \operatorname{erf}(s)+\frac{e^{-s^{2}}}{s \sqrt{\pi}}\right]
\end{aligned}
$$

where $\mathrm{m}, \mathrm{n}, \mathrm{u}$, and $\mathrm{p}$ are the particle mass, number density, bulk velocity, and pressure, respectively. The dimensionless quantity, $\mathrm{s}$, is defined as

$$
\mathrm{s}^{2}=\frac{\mathrm{mnu^{2 }}}{2 \mathrm{p}}
$$

which is the square of the Mach number for a fluid with a specific heat ratio of 2 .

The rapidly decelerating shocked subsolar plasma flow is assumed to be an incompressible fluid (cf. Galeev et al. 1985); consequently $\mathrm{A}(\mathrm{x}) \mathrm{u}(\mathrm{x})=$ constant, where $\mathrm{A}(\mathrm{x})$ is the flow area along the subsolar flow line. Earlier theoretical and experimental studies have shown that behind the shock the implanted ion distribution functions are significantly modified by velocity diffusion and other processes (cf. Ip and Axford 1986, 1987, Gombosi 1987, Kecskeméty et al. 1987). In this region the implanted ion energy spectra above the pickup energy (approximately $10-20 \mathrm{keV}$ ) can be reasonably approximated by exponentials (Kecskeméty et al. 1987). Based on the observations at comets Halley and GiacobiniZinner, the implanted ion loss functions are approximated by expressions (11) through (13). In this approximation, the following equations describe the shocked subsolar flow:

$$
\frac{\partial n_{s w}}{\partial x .}=-\sigma_{p n} n_{n} n_{s w} F\left(s_{s w}\right)
$$

$$
\begin{gathered}
\frac{\partial n_{i}}{\partial x}=s_{i} \\
\frac{\partial u}{\partial x}=-\frac{4 u}{3 p}\left\{m_{i}\left[s_{i}+\sigma_{i n} n_{n} n_{i} F\left(s_{i}\right)\right]+G\right\} \\
\frac{\partial p_{s w}}{\partial x}=-\sigma_{p n} n_{n} p_{s w} H\left(s_{s w}\right)
\end{gathered}
$$

$\frac{\partial p_{i}}{\partial x}=\frac{1}{3} m_{i} u^{2}\left[s_{i}+\sigma_{i n} n_{n} n_{i} F\left(s_{i}\right)\right]-\sigma_{i n} n_{n} p_{i} H\left(s_{i}\right)$

where $x$ is distance along the sun - comet line, $F(s)=I^{(0)} / \mathrm{un}$, $\mathrm{H}(\mathrm{s})=\mathrm{umI}^{(2)} / 3 \mathrm{p}, \mathrm{W}(\mathrm{s})=\mathrm{um}\left(3 \mathrm{I}^{(1)} \mathrm{I}^{(2)}\right) / 4 \mathrm{p}$, and $\mathrm{p}=$ $\left(m_{s w} n_{s w}+m_{i} n_{i}\right)$; while

$$
\begin{gathered}
S_{i}=\frac{n_{n}}{u \tau_{n}}+\sigma_{p n} n_{n} n_{s w} F\left(s_{s w}\right) \\
G=\sigma_{p n} n_{n} \frac{p_{s w}}{u^{2}} W\left(s_{s w}\right)+\sigma_{i n} n_{n} \frac{p_{i}}{u} W\left(s_{i}\right)
\end{gathered}
$$

\section{Results and discussion}

Equations (15) through (19) were solved numerically for the shocked solar wind flow using a set of parameters characterizing comet Halley conditions during the VEGA flyby. The neutral atmospheric number density was approximated by

$$
n_{n}=\frac{Q_{n}}{4 \pi V_{n} r^{2}} \exp \left(-\frac{r}{\lambda_{n}}\right)
$$




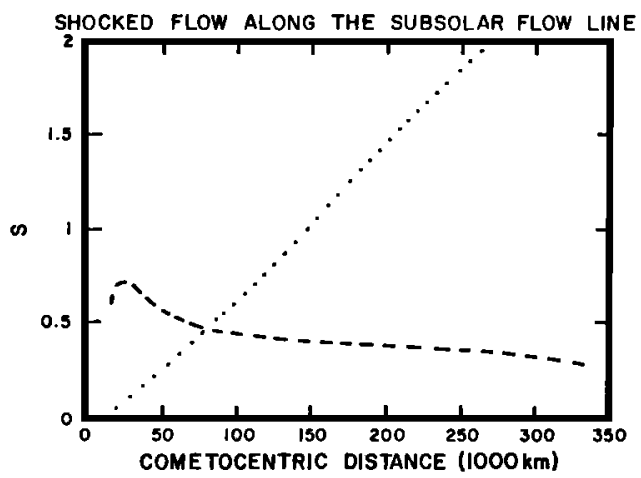

Figure 2. Radial profiles of the dimensionless parameter, s, defined in the text. Dashed lines represent solar wind protons, while dotted lines denote cometary ions.

where $\mathrm{r}$ is the cometocentric distance, while $\mathrm{Q}_{\mathrm{n}}=1.3 \times 10^{30}$ molecules $/ \mathrm{s}, V_{n}=1 \mathrm{~km} / \mathrm{s}$ and $\lambda_{n}=2 \times 10^{6} \mathrm{~km}$. The adopted charge exchange cross sections were $\sigma_{\text {in }}=2 \times 10^{-15} \mathrm{~cm}^{2}$ and $\sigma_{\mathrm{pn}}=2 \times 10^{-15} \mathrm{~cm}^{2}$. The mean molecular mass of the cometary neutrals was assumed to be 16 .

In order to obtain the appropriate initial conditions directly behind the shock, equations (1) through (6) were solved for the unshocked flow, assuming a constant flow area and neglecting charge exchange. In this case the equations can be solved analytically (cf. Galeev et al. 1985). Following the assumptions made by Galeev et al. (1985) a weak shock was assumed at a critical Mach number of $M=2$. Using $n_{\mathrm{sw}}=10$ protons $/ \mathrm{cm}^{3}, T_{s w}=10^{5} \mathrm{~K}, \mathrm{n}_{\mathrm{i}}=0, \mathrm{p}_{\mathrm{i}}=0$ and $\mathrm{u}=500 \mathrm{~km} / \mathrm{s}$ values for the undisturbed solar wind flow, the shock was obtained at $\mathrm{x}_{\mathrm{s}}=3.30 \times 10^{5} \mathrm{~km}$ from the comet. At this point the gas parameters were $\mathrm{u}=146 \mathrm{~km} / \mathrm{s}, \mathrm{n}_{\mathrm{sw}}=34.3, \mathrm{n}_{\mathrm{i}}=0.666$, $\mathrm{p}_{\mathrm{sw}}=1.08 \times 10^{-9} \mathrm{dyn} / \mathrm{cm}^{2}$, and $\mathrm{p}_{\mathrm{i}}=2.57 \times 10^{-8} \mathrm{dyn} / \mathrm{cm}^{2}$. The corresponding heavy ion temperature turned out to be about 24 $\mathrm{keV}$, in good agreement with observations (Kecskeméty et al. 1987).

The solutions of the (15) through (19) differential equation system describing the region between the subsolar shock and the stagnation region are presented in Figure 1. The four panels show the radial variation of the solar wind and implanted ion number densities, flow velocities, pressures, and temperatures, respectively. Inspection of Figure 1 reveals several interesting features of the decelerating flow. Perhaps the most striking one is the rapid transition from a solar wind proton dominated flow to a cometary ion dominated plasma at about $7 \times 10^{4} \mathrm{~km}$. This transition is accompanied by a rapid cooling of the implanted ion population. As demonstrated in the following discussion these changes are caused by a charge exchange "avalanche". It should also be noted that the flow velocity is decreasing almost linearly in the shocked plasma flow and it is reduced to very small values around $2 \times 10^{4} \mathrm{~km}$, where the flow stagnates. In this region the present approximation is no longer valid, because the magnetic field pile-up effects had not been considered in this simplified calculation. The present approximation seems to be reasonably good, however, in the cometopause region, where most of the solar wind protons are replaced by cold heavy cometary ions.

The main physical processes of the cometopause region can be better understood by examining the solar wind continuity equation. In this region $\left(r<<\lambda_{n}\right)$, the exponential decay factor does not play a significant role in the neutral density profile and can be neglected. It can also be seen from Figure 2 that in the shocked plasma flow, $\mathrm{s}_{\mathrm{sw}}$ decreases almost linearly along the sun - comet line. On the other hand, it can be easily shown that the function $F(s)$ can be approximated reasonably well by the following expression:

$$
\mathrm{F}(\mathrm{s})= \begin{cases}1 & \mathrm{~s}>\frac{2}{\sqrt{\pi}} \\ \frac{2}{\mathrm{~s} \sqrt{\pi}} & \mathrm{s} \leq \frac{2}{\sqrt{\pi}}\end{cases}
$$

Using these approximations, the solution of equation (15) is

$\mathrm{n}_{\mathrm{sw}}= \begin{cases}\mathrm{n}_{\mathrm{sw}}\left(\mathrm{r}_{\mathrm{s}}\right) \exp \left[\frac{\left.\mathrm{R}_{0}\left(1-\frac{\mathrm{r}_{\mathrm{s}}}{\mathrm{r}}\right)\right]}{\mathrm{r}} \mathrm{r}>\mathrm{r}_{1}\right. \\ \mathrm{n}_{\mathrm{sw}}\left(\mathrm{r}_{\mathrm{s}}\right) \exp \left[\frac{\mathrm{R}_{0}}{\mathrm{r}_{\mathrm{s}}}-\frac{\mathrm{R}_{0}}{2 \mathrm{r}_{1}}-\frac{\mathrm{R}_{0} \mathrm{r}_{1}}{2 \mathrm{r}^{2}}\right] & \mathrm{r} \leq \mathrm{r}_{1}\end{cases}$

Here $r_{s}$ is the shock distance, $r_{1}$ is the cometocentric distance, where

$$
\mathrm{s}_{\mathrm{sw}}\left(\mathrm{r}_{1}\right)=\frac{2}{\sqrt{\pi}}=\sqrt{\frac{\gamma}{2}} \mathrm{M}_{\mathrm{p}}
$$

where $M_{p}$ is the proton Mach number (ratio of flow velocity and proton acoustic speed). In other words the average thermal velocity of protons becomes equal to the flow velocity at a distance of $r_{1}$. The charge exchange scale length, $R_{0}$, is the following:

$$
\mathrm{R}_{0}=\frac{\sigma_{\mathrm{pn}} \mathrm{Q}_{\mathrm{n}}}{4 \pi \mathrm{V}_{\mathrm{n}}}
$$

The full numerical solution indicates that in our case $r_{1}=1.61 \times 10^{5} \mathrm{~km}$, while an $R_{0}=2.07 \times 10^{4} \mathrm{~km}$ value can be obtained using the adopted parameter values. It should be noted that recently Wallis has also obtained a result similar to expression (24) by assuming a linear radial dependence for the shocked flow velocity (Wallis 1987).

Figure 3 shows a comparison of the numerical solution of the solar wind proton number density and the approximate analytic solution given by expression (24). It can be seen that the two curves track each other quite closely. The proton number density abruptly decreases at a distance of

$$
r_{c}=\sqrt{R_{0} r_{1}}
$$

This sharp charge exchange boundary is the cometopause, which was first observed by the VEGA-2 spacecraft (Gringauz et al. 1986). The subsolar cometopause is about $6 \times 10^{4} \mathrm{~km}$ from the nucleus, which corresponds to a distance of about $1.6 \times 10^{5} \mathrm{~km}$ along the VEGA-2 inbound pass (assuming a 2.15 flaring ratio as suggested by Galeev (1986)),

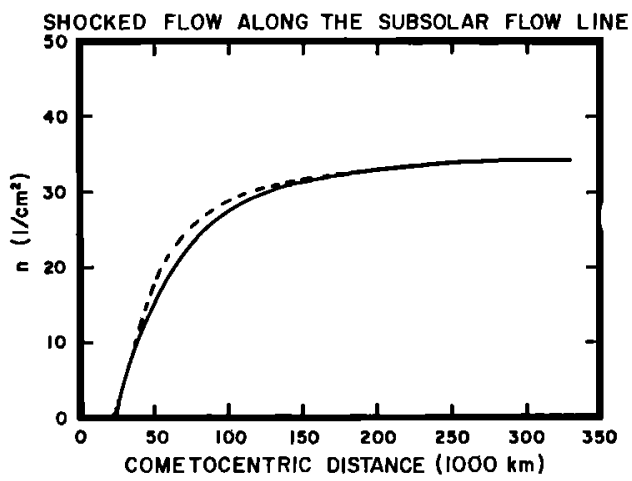

Figure 3. Comparison of solar wind proton number densities obtained by the full numerical solution (solid line) and the analytic approximation given by expression (24) (dotted line). 
which is in good agreement with observations (Gringauz et al. 1986). It is interesting to note that the cometopause distance, $r_{c}$, is the geometric mean of the charge exchange scale length and the characteristic distance, $\mathrm{r}_{1}$ (which is determined by the proton Mach number); therefore it is located significantly further from the nucleus than the collisionopause, which was originally expected to be at a distance of about $R_{0}$ (cf. Mendis et al. 1986). The cometopause thickness obtained from the numerical solution is about $10^{4} \mathrm{~km}$, in good agreement with observations (Gringauz et al. 1986).

The main physical process leading to the formation of the cometopause is the rapid deceleration of the proton flow, which occurs while the temperature remains more or less constant. This process results in a continuous (and almost linear) decrease of the proton Mach number as the flow approaches the nucleus, which in effect means that the ion neutral charge exchange mean free path varies as $r^{3}$; an $r^{2}$ factor comes from the neutral density profile and an additional $r$ dependence is caused by the flow deceleration. This rapid radial variation eventually leads to a charge exchange avalanche, which is observed as the cometopause.

The present calculation has demonstrated that unlike a magnetopause or ionopause the cometopause is not a real plasma boundary, but rather a density crossover point, where the implanted cometary ion density rapidly exceeds the depleted solar wind number density.

\section{Summary}

A two-fluid (solar wind protons and cometary heavy ions) steady-state model has been applied to describe the shocked plasma flow along the subsolar flow line. It was assumed that the proton population followed a Maxwellian velocity distribution, while the cometary ion velocity distribution was isotropic in the plasma frame of reference. Charge exchange and photoionization were considered as the dominant sources.

It was found that the shocked subsolar plasma flow velocity is practically a linear function of the cometocentric distance, $r$, while the proton temperature remains more or less constant. When combined with the $\mathrm{r}^{-2}$ dependence of the neutral density profile, this result predicts an $r^{3}$ dependence for the charge exchange mean free path, resulting in a charge exchange avalanche at the cometopause.

\section{Acknowledgments}

The author is indebted to Drs. T.E. Cravens, A.F. Nagy and M.K. Wallis for illuminating discussions. This work was supported by NSF grant AST-8605994 and NASA grant NGR 23-005-015.

\section{References}

Balsiger, H., Altwegg, K., Bühler, F., Fuselier, S.A., Geiss, J., Goldstein, R., Lazarus, A.J., Meier, A., Neugebauer, M., Rettenmund, U., Rosenbauer, H., Schwenn, R.,
Shelley, E.G., Ungstrup, E., and D.T. Young, The composition and dynamics of cometary ions in the outer coma of Halley, in Proc. 20th ESLAB Symposium on the Exploration of Halley's Comet (eds. B. Battrick, E.J. Rolfe and R. Reinhard), ESA SP-250, Vol. 1., p.99, 1986.

Galeev, A.A., Theory and observations of solar wind/cometary plasma interaction processes, in Proc. 20th ESLAB Symposium on the Exploration of Halley's Comet (eds. B. Battrick, E.J. Rolfe and R. Reinhard), ESA SP250, Vol. 1., p.3, 1986.

Galeev, A.A., Cravens, T.E., and Gombosi, T.I., Solar wind stagnation near comets, Astrophys. J., 289, 807, 1985.

Gombosi, T.I., Preshock region acceleration of implanted cometary $\mathrm{H}^{+}$and $\mathrm{O}^{+}$, J. Geophys. Res., in press, 1987.

Gringauz K.I., Gombosi T.I., Tátrallyay M., Verigin M.I., Remizov A.P., Richter A.K., Apáthy I., Szemerey I., Dyachkov A.V., Balakina O.V., Nagy A.F.: VEGA observations of the cometopause and cometary plasma region, Geophys. Res. Lett, 13, 613-616, 1986.

Ip, W.-H., and Axford, W.I., The acceleration of particles in the vicinity of comets, Planet. Space Sci., 34, 1061, 1986.

Ip, W.-H., and Axford, W.I., A numerical simulation of charged particle acceleration and pitch-angle scattering in the turbulent plasma environment of comet Halley, Proc. 20th International Cosmic Ray Conference, in press, 1987.

Kecskeméty K., Cravens T.E., Afonin V.V., Erdös G., Eroshenko E.G., Gan L., Gombosi T.I., Gringauz K.I., Keppler E., Klimenko I.N., Marsden R.G., Nagy A.F., Remizov A.P., Richter A.K., Riedler W., Schwingenschuh K., Somogyi A.J., Szegö K., Tátrallyay M., Varga A., Verigin M.I., Wenzel K.P., Pick-up ions in the unshocked solar wind at comet Halley, J. Geophys. Res., submitted, 1987.

Mendis, D.A., Smith, E.J., Tsurutani, B.T., Slavin, J.A. Jones, D.E., and Siscoe, G.L., Comet - solar wind interaction: Dynamical length scales and models, Geophys. Res. Lett., 13, 239, 1986.

Reme, H., Sauvaud, J.A., d'Uston, C., Cros, A., Anderson, K.A., Carlson, C.W., Curtis, D.W., Lin, R.P., Korth, A., Richter, A.K., and D.A. Mendis, General features of the comet Halley - solar wind interaction form plasma observations, in Proc. 20th ESLAB Symposium on the Exploration of Halley's Comet (eds. B. Battrick, E.J. Rolfe and R. Reinhard), ESA SP-250, Vol. 1., p.29, 1986.

Wallis, M.K., Plasma transition layers in Halley's comet, paper presented at the International Conference on Cometary Plasma Physics, Trieste, Italy, June 9-11, 1987.

T.I. Gombosi, The University of Michigan, Department of Atmospheric and Oceanic Science, Space Physics Research Laboratory, Ann Arbor, Michigan 48109-2143.

(Received September 8, 1987; accepted October 2, 1987.) 